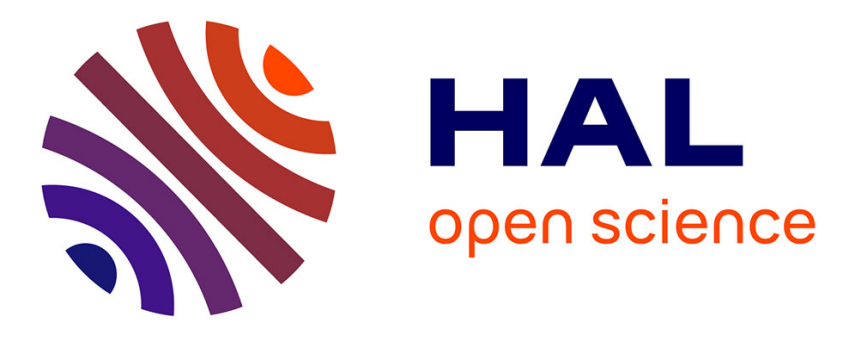

\title{
Emission and its back-reaction accompanying electron motion in relativistically strong and QED-strong pulsed laser fields
}

Igor V. Sokolov, John A. Nees, Victor P. Yanovsky, Natalia Naumova, Gérard

A. Mourou

\section{To cite this version:}

Igor V. Sokolov, John A. Nees, Victor P. Yanovsky, Natalia Naumova, Gérard A. Mourou. Emission and its back-reaction accompanying electron motion in relativistically strong and QED-strong pulsed laser fields. Physical Review E : Statistical, Nonlinear, and Soft Matter Physics, 2010, 81 (3), pp.036412.1-036412.13. 10.1103/physreve.81.036412 . hal-00838485

HAL Id: hal-00838485

https://hal-ensta-paris.archives-ouvertes.fr/hal-00838485

Submitted on 7 Dec 2015

HAL is a multi-disciplinary open access archive for the deposit and dissemination of scientific research documents, whether they are published or not. The documents may come from teaching and research institutions in France or abroad, or from public or private research centers.
L'archive ouverte pluridisciplinaire HAL, est destinée au dépôt et à la diffusion de documents scientifiques de niveau recherche, publiés ou non, émanant des établissements d'enseignement et de recherche français ou étrangers, des laboratoires publics ou privés. 


\title{
Emission and its back-reaction accompanying electron motion in relativistically strong and QED-strong pulsed laser fields
}

\author{
Igor V. Sokolov, ${ }^{1} *$ John A. Nees, ${ }^{2}$ Victor P. Yanovsky, ${ }^{2}$ Natalia M. Naumova, ${ }^{3}$ and Gérard A. Mourou ${ }^{4}$ \\ ${ }^{1}$ Space Physics Research Laboratory, University of Michigan, Ann Arbor, Michigan 48109, USA \\ ${ }^{2}$ Center for Ultrafast Optical Science and FOCUS Center, University of Michigan, Ann Arbor, Michigan 48109, USA \\ ${ }^{3}$ Laboratoire d'Optique Appliquée, UMR 7639 ENSTA, Ecole Polytechnique, CNRS, 91761 Palaiseau, France \\ ${ }^{4}$ Institut de la Lumière Extrême, UMS 3205 ENSTA, Ecole Polytechnique, CNRS, 91761 Palaiseau, France \\ (Received 8 October 2009; revised manuscript received 11 December 2009; published 25 March 2010)
}

\begin{abstract}
The emission from an electron in the field of a relativistically strong laser pulse is analyzed. At pulse intensities of $J \geq 2 \times 10^{22} \mathrm{~W} / \mathrm{cm}^{2}$ the emission from counterpropagating electrons is modified by the effects of quantum electrodynamics (QED), as long as the electron energy is sufficiently high: $\mathcal{E} \geq 1 \mathrm{GeV}$. The radiation force experienced by an electron is for the first time derived from the QED principles and its applicability range is extended toward the QED-strong fields.
\end{abstract}

DOI: 10.1103/PhysRevE.81.036412

PACS number(s): 52.38.Ph, 41.60.- m, 12.20.Ds

\section{INTRODUCTION}

\section{A. QED-strong fields}

In quantum electrodynamics (QED) an electric field, $E$, should be treated as strong if it exceeds the Schwinger limit: $E \geq E_{S}$, where

$$
E_{S}=\frac{m_{e} c^{2}}{|e| \chi_{C}}
$$

(see [1]). Such field is potentially capable of separating a virtual electron-positron pair providing an energy, which exceeds the electron rest mass energy, $m_{e} c^{2}$, to a charge, $e=$ $-|e|$, over an acceleration length as small as the Compton wavelength,

$$
\chi_{C}=\frac{\hbar}{m_{e} c} \approx 3.9 \times 10^{-11} \mathrm{~cm} .
$$

Spatial scales associated with the field should be greater than $x_{C}$.

Typical effects in QED-strong fields are: electron-positron pair creation from high-energy photons, high-energy photon emission from electrons or positrons, and electron-positron cascade development (see [2,3]) as the result of the first two processes.

Less typical and often forbidden by conservation laws is direct pair separation from vacuum. This effect may only be significant if the field invariants as defined in [4], $F_{1}$ $=(\mathbf{B} \cdot \mathbf{E})$ and $F_{2}=E^{2}-B^{2}$, are large enough. Indeed, the considerations relating to pair creation are applicable only in the frame of reference in which $B=0$ or $\mathbf{B} \| \mathbf{E}$. The electric field in this frame of reference, $E_{0}^{2}=F_{2} / 2+\sqrt{F_{1}^{2}+F_{2}^{2} / 4}$, exceeds the Schwinger limit only if the field invariants are sufficiently large.

Here the case of weak field invariants is considered:

\footnotetext{
*igorsok@umich.edu
}

$$
\left|F_{1}\right| \ll E_{S}^{2}, \quad\left|F_{2}\right| \ll E_{S}^{2},
$$

and any corrections of the order of $F_{1} / E_{S}^{2}$ and $F_{2} / E_{S}^{2}$ are neglected (see [5] about such corrections). So, neither the cases when the field itself is too strong nor the cases when its spatial scale is too short are considered here. Below, the term "strong field" is only applied to the field experienced by a particle (electron or positron).

Particularly, a QED-strong electric field,

$$
E_{0}=\frac{|\mathbf{p} \times \mathbf{B}|}{m_{e} c},
$$

may be exerted on relativistic charged particles with momentum, $\mathbf{p}$, gyrating in the strong magnetic field, $\mathbf{B}$, of a neutron star, as the result of the Lorentz transformation of the electromagnetic field. The field as in Eq. (2) may exceed the Schwinger limit as long as $|\mathbf{p}| \gg m_{e} c$ and/or the magnetic field is strong enough.

\section{B. QED-strong laser fields}

In a laboratory experiment QED-strong fields may be created in the focus of an ultrabright laser. Consider QED effects in a relativistically strong pulsed field [2]:

$$
\sqrt{\mathbf{a}^{2}} \gg 1, \quad \mathbf{a}=\frac{e \mathbf{A}}{m_{e} c^{2}},
$$

with $\mathbf{A}$ being the vector potential of the wave. In the laboratory frame of reference the electric field is not QED strong for achieved laser intensities $J \sim 10^{22} \mathrm{~W} / \mathrm{cm}^{2}[6]$ and even for the $J \sim 10^{25} \mathrm{~W} / \mathrm{cm}^{2}$ intensity projected [7]. Moreover, both field invariants vanish for one-dimensional (1D) waves, reducing the probability of direct pair creation from vacuum by virtue of the laser field's proximity to $1 \mathrm{D}$ wave.

Nonetheless, a counterpropagating particle in a 1D wave, $\mathbf{a}(\xi), \xi=\omega t-(\mathbf{k} \cdot \mathbf{x})$, may experience a QED-strong field, $E_{0}$ $=|d \mathbf{A} / d \xi| \omega\left(\mathcal{E}-p_{\|}\right) / c$, because the laser frequency, $\omega$, is Doppler upshifted in the frame of reference comoving with the electron. Herewith the electron dimensionless energy, $\mathcal{E}$, and its momentum are related to $m_{e} c^{2}$ and $m_{e} c$, correspondingly, 
and subscript $\|$ herewith denotes the vector projection on the direction of the wave propagation. The Lorentz-transformed field exceeds the Schwinger limit if

$$
\chi \sim E_{0} / E_{S}=\frac{\chi_{C}}{\chi}\left(\mathcal{E}-p_{\|}\right)\left|\frac{d \mathbf{a}}{d \xi}\right| \gg 1,
$$

where $\chi=c / \omega$. Note that the above-mentioned restriction on the field spatial scale is here assumed to be fulfilled for the upshifted wave frequency:

$$
\omega\left(\mathcal{E}-p_{\|}\right) \ll c / \chi_{C} .
$$

Nevertheless, the condition as in Eq. (4) may be fulfilled as long as the field is strong enough. Numerical values of the parameter, $\chi$, may be conveniently expressed in terms of the local instantaneous (not time-average) intensity of the laser wave, $J$ :

$$
\chi=\frac{3}{2} \frac{\chi_{C}}{\chi}\left(\mathcal{E}-p_{\|}\right)\left|\frac{d \mathbf{a}}{d \xi}\right| \approx 0.7 \frac{\left(\mathcal{E}-p_{\|}\right)}{10^{3}} \sqrt{\frac{J}{10^{23}\left[\mathrm{~W} / \mathrm{cm}^{2}\right]}},
$$

the choice of the numerical factor of $3 / 2$ is explained below. For a counterpropagating electron of energy $\sim 1 \mathrm{GeV}$, that is, for $\left(\mathcal{E}-p_{\|}\right) \sim 4 \times 10^{3}$, the QED-strength parameter is greater than one even with the laser intensities already achieved.

The condition of $\chi>1$ also separates the parameter range of the Compton effect from that of the Thomson effect under the condition of Eq. (3). The distinctive feature of the Compton effect is an electron recoil, which is significant, if a typical emitted photon energy, $\hbar \omega_{c}$, is comparable with the electron energy [8]. Their ratio, $\chi=\chi_{C} \omega_{c} /(c \mathcal{E})$, equals $\chi$ as defined in Eq. (7) with the proper numerical factors [cf. Eq. (18)]. It should be noted, however, that under the conditions discussed in Eqs. (3) and (5) the Compton effect drastically changes.

\section{Classical radiation loss rate as an input parameter for QED}

Radiation processes in QED-strong fields are entirely controlled by the local value of $E_{0}$ (this statement may be found in [9], Sec. 101). A good signature for $E_{0}$ is the radiation loss rate of a charge as introduced in the classical electrodynamics:

$$
I_{\mathrm{cl}}\left(E_{0}\right)=\frac{2 e^{4} E_{0}^{2}}{3 m_{e}^{2} c^{3}}=-\frac{2 e^{2} f^{\mu} f_{\nu}}{3 m_{e}^{2} c^{3}},
$$

which is a Lorentz invariant. Therefore, it may be expressed in any frame of reference in terms of a four-square of the Lorentz four-force, $f^{\mu}=\left(f^{0}, \mathbf{f}\right)=\mathcal{E}\left(\mathbf{f}^{(3)} \cdot \mathbf{v} / c, \mathbf{f}^{(3)}\right)$, where $\mathbf{v}$ is the velocity vector and

$$
\begin{gathered}
\mathbf{f}^{(3)}=e \mathbf{E}+\frac{\mathrm{e}}{c}[\mathbf{v} \times \mathbf{B}], \\
\left.f^{0}=e \mathbf{E} \cdot \mathbf{p}, \quad \mathbf{f}=e \mathcal{E} \mathbf{E}+e[\mathbf{p} \times \mathbf{B}]\right) .
\end{gathered}
$$

So, the QED-strength of the field may be determined in evaluating $I_{\mathrm{cl}}$ and its ratio to $I_{C}=I_{\mathrm{cl}}\left(2 E_{S} / 3\right)$ :

$$
\chi=\sqrt{\frac{I_{\mathrm{cl}}}{I_{C}}}, \quad I_{C}=\frac{8 e^{2} c}{27 \chi_{C}^{2}} .
$$

If $\chi \geq 1$ then the actual radiation loss rate differs from $I_{\mathrm{cl}}$, however, it may be recalculated using $I_{\mathrm{cl}}$ as a sole input parameter.

\section{Possible realization of QED-strong fields in laboratory experiments}

The first experiments which demonstrated QED effects in a laser field were fulfilled using an electron beam of energy $\approx 46.6 \mathrm{GeV}$ (see [10]), which interacted with a counterpropagating terawatt laser pulse of intensity $J$ $\sim 10^{18} \mathrm{~W} / \mathrm{cm}^{2}$. A reasonably high value of $\chi \approx 0.4$ had been achieved, however, the laser field was not relativistically strong with $|\mathbf{a}| \leq 1$. The high value of $\chi$ had been achieved at the cost of very high energy of the upshifted laser wave: the transformed photon energy amounted to $\sim 0.1 \mathrm{MeV}$, which is not small as compared to the electron rest mass energy, $m_{e} c^{2} \approx 0.51 \mathrm{MeV}$. It could be interesting to upgrade this experiment toward the highest achievable laser intensities $\geq 2$ $\times 10^{22}$ with the use of a wakefield-accelerated beam of electrons of energy $\sim 1 \mathrm{GeV}$ (see [11]). First, the 2-3 times larger value of the QED parameter, $\chi$, may be achieved with the exponentially increased probability for pair creation. Second, such experiment would be highly relevant to the processes which will occur in the course of laser-plasma interaction at even stronger laser intensities.

Indeed, counterpropagating electrons can be generated while a laser pulse is interacting with a solid target. For this reason, the radiation effects in the course of laser-plasma interaction are widely investigated (see $[8,12]$ ). With future progress in laser technology and by achieving intensities of $J \sim 10^{23} \mathrm{~W} / \mathrm{cm}^{2}$ laser-plasma interactions will be strongly modified by QED effects so that the capability to model these effects now is of interest.

\section{E. Radiation back-reaction}

The principle matter in this paper is an account of the radiation back-reaction acting on a charged particle. The radiation losses reduce the particle energy, affecting both the particle motion and the radiation losses themselves.

This effect can be consistently described by solving the dynamical equation which appears to reduce to the modified Lorentz-Abraham-Dirac equation as derived in $[13,14]$. The particular element addressed here is that in this equation the radiation back-reaction on the electron motion should be expressed in terms of the emission probability, while applied to QED-strong fields.

In Sec. II the emission from an electron in the field of a relativistically strong wave is discussed within the framework of classical electrodynamics. The transition from the vector amplitude of emission as described in [15] to the instantaneous spectrum of emission is treated in terms of the formation time, a concept, which is not often used in classical electrodynamics. For QED-strong laser pulses the calculation of the emission probability is given in Sec. III. The radiation processes in QED-strong fields appear to be reduc- 
ible to a frequency downshift in the classical vector amplitude of emission resulting from the electron recoil, accompanied by a contribution to emission associated with the magnetic moment of electron. The radiation effect on the electron motion in strong fields is discussed in Sec. IV. The conclusion summarizes the results and discusses future prospectives.

\section{EMISSION IN RELATIVISTICALLY STRONG FIELDS}

In this section QED effects are not yet considered, but the electromagnetic wave field is assumed to be relativistically strong. Angular and frequency distributions of electron emission are discussed. The goal is to establish a connection between the methods usually applied to calculate emission in weaker fields, on one hand, and the conceptually different QED approach on the other. For relativistically strong laser fields, even though QED effects do not yet come into a power, still some concepts of the QED emission theory appear to be applicable and useful, among them are the formation time of emission and instantaneous spectrum of emission.

In weaker fields, especially for the particular case of a harmonic wave, the emitted power is given by an integral over many periods of the wave. This standard approach, however, may become meaningless as applied to the ultrastrong laser pulses for many reasons. These pulses may be so short that they cannot be thought of as harmonic waves. Their fields may be strong enough to force an electron to expend its energy on radiation faster than a single wave period. However, an even more important point is that the radiation loss rate and even the spectrum of radiation is no longer an integral characteristic of the particle motion through a number of wave periods: a local dependence of emission on both particle and field characteristics is typical for the strong fields.

\section{A. Transformed space-time}

A method facilitating many derivations involves the introduction of a specific space-time coordinate frame. Consider a 1D wave field taken in the Lorentz calibration:

$$
a^{\mu}=a^{\mu}(\xi), \quad \xi=(k \cdot x), \quad(k \cdot a)=0,
$$

with $a^{\mu}=(0, \mathbf{a}), k^{\mu}$, and $x^{\mu}$ being the four-vectors of the potential, the wave, and the coordinates. Herewith the four-dot product is introduced in a usual manner:

$$
(k \cdot x)=k^{\mu} x_{\mu}=\omega t-(\mathbf{k} \cdot \mathbf{x}),
$$

etc. Spacelike three-vectors (i.e., the first to the third components of a four-vector) in contrast with four-vectors are denoted in bold, four-indices are denoted with Greek letters. Note that a metric signature $(+,-,-,-)$ is used, therefore, for spacelike vectors the three-dimensional (3D) scalar product and four-dot product have opposite signs, particularly:

$$
\left(\frac{d \mathbf{a}}{d \xi}\right)^{2}=-\left(\frac{d a}{d \xi}\right)^{2} \geq 0 .
$$

We introduce a transformed space-time (TST):

$$
x^{0,1}=\left(c t \mp x_{\|}\right) / \sqrt{2}, \quad x^{2,3}=\mathbf{x}_{\perp},
$$

subscript $\perp$ denoting the vector components orthogonal to $\mathbf{k}$. The properties of the TST provide a convenient description for the classical motion of an electron in the $1 \mathrm{D}$ wave field. First, note that

$$
d x^{0}=\frac{\chi d \xi}{\sqrt{2}}, \quad p^{0}=\frac{\chi(k \cdot p)}{\sqrt{2}}, \quad(p \cdot k)=\frac{\mathcal{E}-p_{\|}}{\chi} .
$$

Second, the generalized momentum components, $p^{0}$ and $\mathbf{p}_{\perp 0}=\mathbf{p}_{\perp}+\mathbf{a}$, are conserved. Third, the metric tensor in the TST is

$$
G^{01}=G^{10}=1, \quad G^{22}=G^{33}=-1, \quad G^{\mu \nu}=G_{\mu \nu} .
$$

Finally, the identity, $\mathcal{E}^{2}=p^{2}+1$, being expanded in the TST metric gives

$$
p^{1}=\frac{1+\mathbf{p}_{\perp}^{2}}{2 p^{0}}=\frac{1+\left(\mathbf{p}_{\perp 0}-\mathbf{a}\right)^{2}}{\sqrt{2} \chi(k \cdot p)} .
$$

The classical radiation loss rate is found by virtue of expanding the Lorentz force squared in the TST:

$$
I_{\mathrm{cl}}=-\frac{2 e^{2}}{3 c} \frac{(f \cdot f)}{m_{e}^{2} c^{2}}=\frac{2 e^{2} c(k \cdot p)^{2}}{3}\left(\frac{d \mathbf{a}}{d \xi}\right)^{2} .
$$

The derivative over $x^{0}$ or, the same, over $\xi$ is conveniently related to the derivative over the proper time for electron:

$$
\frac{d}{d \tau}=\mathcal{E}\left[\frac{\partial}{\partial t}+\left(\mathbf{v} \cdot \frac{\partial}{\partial \mathbf{x}}\right)\right]=c(k \cdot p) \frac{d}{d \xi} .
$$

\section{B. Classical trajectory and momenta retarded product}

Many characteristics of emission may be expressed in terms of the relationship between the four-momenta of the electron at different instants:

$$
p^{\mu}(\xi)=p^{\mu}\left(\xi^{\prime}\right)-\delta a^{\mu}+\frac{2\left[p\left(\xi^{\prime}\right) \cdot \delta a\right]-(\delta a)^{2}}{2(k \cdot p)} k^{\mu},
$$

where

$$
\delta a^{\mu}=a^{\mu}(\xi)-a^{\mu}\left(\xi^{\prime}\right) .
$$

As a consequence from Eq. (10), one can obtain the expression for the momenta retarded product (MRP):

$$
\left[p(\xi) \cdot p\left(\xi^{\prime}\right)\right]=1-\frac{(\delta a)^{2}}{2}=1+\frac{(\delta \mathbf{a})^{2}}{2} .
$$

Note that the MRP is given by Eq. (11) for an arbitrary difference between $\xi$ and $\xi^{\prime}$, but only for the particular case of the 1D wave field. However the limit of this formula as $\left|\xi-\xi^{\prime}\right| \rightarrow 0$, which is as follows:

$$
\left.\left[p(\xi) \cdot p\left(\xi^{\prime}\right)\right]\right|_{\left|\xi-\xi^{\prime}\right| \rightarrow 0} \approx 1+\frac{1}{2}\left(\xi-\xi^{\prime}\right)^{2}\left|\frac{d \mathbf{a}}{d \xi}\right|^{2}
$$

or, in terms of the MRP in the proper time, $\tau$ : 


$$
[p(\tau) \cdot p(\tau+\delta \tau)]=1-(\delta \tau)^{2} \frac{(f \cdot f)}{2 m_{e}^{2} c^{2}},
$$

has a much wider range of applicability. Equation (12) is derived from the equation of motion:

$$
\frac{d p^{\mu}}{d \tau}=\frac{f^{\mu}}{m_{e} c},
$$

using the identities:

$$
\begin{gathered}
{[p(\tau) \cdot p(\tau)]=1, \quad[p(\tau) \cdot f(\tau)]=0,} \\
\frac{d[p(\tau) \cdot p(\tau+\delta \tau)]}{d(\delta \tau)}=-\delta \tau\left(\frac{d p}{d \tau} \cdot \frac{d p}{d \tau}\right)+O\left((\delta \tau)^{2}\right) .
\end{gathered}
$$

\section{Vector amplitude of emission in classical electrodynamics}

In Ref. [15] the frequency spectrum and angular distribution, $d R_{\mathrm{cl}} /\left(d \omega^{\prime} d \mathbf{n}\right)$, of the radiation energy, $d R_{\mathrm{cl}}$, emitted by an electron and related to the interval of frequency, $d \omega^{\prime}$, and to the element of solid angle, $d \mathbf{n}$, for a polarization vector, $\mathbf{l}$, is described with the following formula:

$$
\frac{d R_{\mathrm{cl}}}{d \omega^{\prime} d \mathbf{n}}=\frac{\left(\omega^{\prime}\right)^{2}}{4 \pi^{2} c}\left|\left(\mathbf{A}_{\mathrm{cl}}\left(\omega^{\prime}\right) \cdot \mathbf{l}^{*}\right)\right|^{2} .
$$

Here the superscript asterisk means the complex conjugation and the vector amplitude of emission, $\mathbf{A}_{\mathrm{cl}}\left(\omega^{\prime}\right)$, is given by the following equation:

$$
\mathbf{A}_{\mathrm{cl}}\left(\omega^{\prime}, \mathbf{n}\right)=\frac{e}{c} \int_{-\infty}^{+\infty} \mathbf{v}(t) \exp \left(i \omega^{\prime}\left\{t-\frac{[\mathbf{n} \cdot \mathbf{r}(t)]}{c}\right\}\right) d t,
$$

see Eq. (14.67) in Ref. [15] followed by the discussion of the way to account for a polarization. The use of the same notation, $\mathbf{A}$, both for the emission vector amplitude and for the vector potential should not mislead the reader. Recall that the emission vector amplitude is closely related to the Fouriertransformed vector potential in the far-field zone of emission.

We introduce a four-vector amplitude of emission,

$$
A_{\mathrm{cl}}^{\mu}\left(\omega^{\prime}, \mathbf{n}\right)=e \int_{-\infty}^{+\infty} p^{\mu}(\tau) \exp \left\{i c \int^{\tau}\left[k^{\prime} \cdot p\left(\tau^{\prime}\right)\right] d \tau^{\prime}\right\} d \tau,
$$

which is expressed in terms of the proper time for the electron and its (dimensionless) four-momentum. As long as $\left(k^{\prime} \cdot p\right) / c$ is a frequency of the emitted photon in the frame of reference comoving with the electron, the four-vector amplitude is the Fourier integral of the electron four-momentum with the Lorentz-modified frequency.

Note the following properties of the four-vector amplitude. First, its spacelike vector components, which are perpendicular to the wave vector of the emitted photon, coincide with those for three-vector amplitude, hence, they quantify the polarization properties of the emission for two different polarizations. Second, the dot product, $\left(A_{\mathrm{cl}} \cdot k^{\prime}\right)$, vanishes as being the integral of a perfect time derivative. Now construct the dot product $\left(A_{\mathrm{cl}} \cdot A_{\mathrm{cl}}^{*}\right)$ and expand it in the TST, which is formulated in terms of the emitted wave:

$$
\left(A_{\mathrm{cl}} \cdot A_{\mathrm{cl}}^{*}\right)=A_{\mathrm{cl}}^{0}\left(A_{\mathrm{cl}}^{1}\right)^{*}+A_{\mathrm{cl}}^{1}\left(A_{\mathrm{cl}}^{0}\right)^{*}-\left|A_{\mathrm{cl}}^{2}\right|^{2}-\left|A_{\mathrm{cl}}^{3}\right|^{2} .
$$

From the above properties of the four-vector amplitude, the first two terms vanish identically as $A_{\mathrm{cl}}^{0} \propto\left(A_{\mathrm{cl}} \cdot k^{\prime}\right)=0$. The other two terms as taken with the proper factor give the emitted energy summed up over polarizations, therefore, the latter sum may be expressed as follows:

$$
\sum_{\mathbf{l}} \frac{d R_{\mathrm{cl}}}{d \omega^{\prime} d \mathbf{n}}=-\frac{\left(\omega^{\prime}\right)^{2}}{4 \pi^{2} c}\left(A_{\mathrm{cl}} \cdot A_{\mathrm{cl}}^{*}\right) .
$$

Now we introduce the radiation loss rate $d I_{\mathrm{cl}} /\left(d \omega^{\prime} d \mathbf{n}\right)$ related to the unit of time, the element of a solid angle, and the frequency interval. Its connection to $d R_{\mathrm{cl}} /\left(d \omega^{\prime} d \mathbf{n}\right)$ is evident:

$$
\sum_{\mathrm{l}} \frac{d R_{\mathrm{cl}}}{d \omega^{\prime} d \mathbf{n}}=\int_{-\infty}^{+\infty} \frac{d I_{\mathrm{cl}}(t)}{d \omega^{\prime} d \mathbf{n}} d t=\int_{-\infty}^{+\infty} \frac{d I_{\mathrm{cl}}(\tau)}{d \omega^{\prime} d \mathbf{n}} \mathcal{E}(\tau) d \tau .
$$

In Eq. (14) the dot product of the four-vector amplitudes $\left(A_{\mathrm{cl}} \cdot A_{\mathrm{cl}}^{*}\right)$ is in fact the product of two integrals over $d \tau$, which can be represented as the double integral, over, say, $d \tau_{1} d \tau_{2}$. We transform the integration variables in this double integral by introducing $\tau=\left(\tau_{1}+\tau_{2}\right) / 2, \theta=\tau_{1}-\tau_{2}$. The spectral and angular distribution of the radiation loss rate may be expressed in terms of the Fourier integral of the MRP:

$$
\begin{aligned}
\frac{d I_{\mathrm{cl}}(\tau)}{d \omega^{\prime} d \mathbf{n}}= & -\frac{e^{2}\left(\omega^{\prime}\right)^{2}}{4 \pi^{2} c \mathcal{E}(\tau)} \int_{-\infty}^{+\infty}\left[p\left(\tau+\frac{\theta}{2}\right) \cdot p\left(\tau-\frac{\theta}{2}\right)\right] \\
& \times \exp \left\{i c \int_{\tau-\theta / 2}^{\tau+\theta / 2}\left[k^{\prime} \cdot p\left(\tau^{\prime}\right)\right] d \tau^{\prime}\right\} d \theta .
\end{aligned}
$$

\section{Frequency spectrum and formation time}

The specific feature of the particle relativistic motion in strong laser fields is that the main contribution to the above integral comes from a brief time interval with small values of $\theta$. A closely related point is that the emitted radiation is abruptly beamed about the direction of the velocity vector, $\mathbf{p}(\tau) /|\mathbf{p}(\tau)|$. Therefore, in the following expansion of the frequency in the comoving frame:

$\left[k^{\prime} \cdot p\left(\tau^{\prime}\right)\right]=\left\{\left[k^{\prime}-\frac{\omega^{\prime}}{c \mathcal{E}(\tau)} p(\tau)\right] \cdot p\left(\tau^{\prime}\right)\right\}+\frac{\omega^{\prime}}{c \mathcal{E}}\left[p(\tau) \cdot p\left(\tau^{\prime}\right)\right]$,

in the first dot product one may approximate $p^{\mu}\left(\tau^{\prime}\right) \approx p^{\mu}(\tau)$. Indeed, for an ultrarelativistic electron and a photon, which both propagate in the same direction, the difference between $p^{\mu} / \mathcal{E}$ and $c\left(k^{\prime}\right)^{\mu} / \omega^{\prime}$ is already small, therefore in the second multiplier of the dot product the small difference, $p^{\mu}(\tau)$ $-p^{\mu}\left(\tau^{\prime}\right)$, may be neglected:

$$
\begin{aligned}
c \int_{\tau-\theta / 2}^{\tau+\theta / 2}\left[k^{\prime} \cdot p\left(\tau^{\prime}\right)\right] d \tau^{\prime} \approx & \theta c\left[k^{\prime} \cdot p(\tau)\right] \\
& +\frac{\omega^{\prime}}{\mathcal{E}} \int_{\tau-\theta / 2}^{\tau+\theta / 2}\left\{\left[p(\tau) \cdot p\left(\tau^{\prime}\right)\right]-1\right\} d \tau^{\prime} .
\end{aligned}
$$

Now the only angle-dependent multiplier is 
$\exp \left\{-i \theta c\left[k^{\prime} \cdot p(\tau)\right]\right\}$. For simplicity, the angular spectrum of emission can be approximated with the Dirac function:

$$
\frac{d I_{\mathrm{cl}}(\tau)}{d \omega^{\prime} d \mathbf{n}}=\delta^{2}\left(\mathbf{n}-\frac{\mathbf{p}}{|\mathbf{p}|}\right) \frac{d I_{\mathrm{cl}}(\tau)}{d \omega^{\prime}},
$$

and with the use of the formula (see Sec. 90 in Ref. [9])

$$
\int \exp \left\{i \theta c\left[k^{\prime} \cdot p(\tau)\right]\right\} d \mathbf{n}=\frac{2 \pi i}{\omega^{\prime} \mathcal{E}(\tau) \theta} \exp \left[\frac{i \omega \theta}{2 \mathcal{E}(\tau)}\right],
$$

the following expression may be found for the frequency spectrum of emission:

$$
\begin{aligned}
\frac{d I_{\mathrm{cl}}(\tau)}{d \omega^{\prime}}= & \frac{e^{2} \omega^{\prime}}{2 \pi c \mathcal{E}^{2}(\tau)} \int_{-\infty}^{+\infty} \frac{1}{\theta}\left[p\left(\tau+\frac{\theta}{2}\right) \cdot p\left(\tau-\frac{\theta}{2}\right)\right] \\
& \times \sin \left[\frac{\omega^{\prime}}{\mathcal{E}(\tau)}\left(\frac{\theta}{2}+\int_{\tau-\theta / 2}^{\tau+\theta / 2}\left\{\left[p(\tau) \cdot p\left(\tau^{\prime}\right)\right]-1\right\} d \tau^{\prime}\right)\right] d \theta .
\end{aligned}
$$

Thus, the frequency spectrum of emission is entirely determined by the MRP, which is a scalar Lorentz-invariant function of the proper time. Both the fore-exponential factor and the argument of the exponential function depend on the mentioned MRP. Therefore, both the spectral composition of the MRP and its magnitude may be of importance. Their relative role is controlled by the ratio of the frequency of the electron motion, $\omega_{0}$, to the acceleration magnitude, both being determined in the comoving frame of reference. Here the field is assumed to be so strong that the acceleration it causes plays the dominant role, i.e., the following inequality is claimed:

$$
-\frac{(f \cdot f)}{m_{e}^{2} c^{2}} \gg \omega_{0}^{2}
$$

Under these circumstances, the integral determining the emission spectrum is calculated by virtue of the displacement of the integration contour in the plane of the complex variable, $\theta$, so that the deformed contour passes through the point of a stationary phase, $\theta_{\mathrm{st}}$. In this "saddle" point the phase gradient turns to zero:

$$
\frac{d}{d \theta}\left(\frac{\theta}{2}+\int_{\tau-\theta / 2}^{\tau+\theta / 2}\left\{\left[p(\tau) \cdot p\left(\tau^{\prime}\right)\right]-1\right\} d \tau^{\prime}\right)=0 .
$$

The larger the acceleration becomes, the closer the stationary phase point, $\theta_{\mathrm{st}}$ draws to the real axis, and hence, the shorter the time interval becomes, $\theta \sim \theta_{\mathrm{f}}=\left|\theta_{\mathrm{st}}\right|$, which gives the nonvanishing contribution to the emission spectrum. The characteristic duration of this time interval $\theta_{\mathrm{f}}=\left|\theta_{\mathrm{st}}\right|$ is referred to as a formation time (or coherence time-see $[16,17]$ ). At the limit of large accelerations the formation time is given by the following formula:

$$
\theta_{\mathrm{st}}= \pm i \frac{2 m_{e} c}{\sqrt{-(f \cdot f)}}, \quad \theta_{\mathrm{f}}=\frac{2 m_{e} c}{\sqrt{-(f \cdot f)}}
$$

where the approximation for the MRP as in Eq. (12) is applied at $|\theta| \leq \theta_{\mathrm{f}}$. With the use of Eq. (12) the universal emission spectrum is obtained:

$$
\begin{aligned}
\frac{d I_{\mathrm{cl}}(\tau)}{d \omega^{\prime}}= & \frac{e^{2} \omega^{\prime}}{2 \pi c \mathcal{E}^{2}(\tau)} \int_{-\infty}^{+\infty}\left\{\frac{1}{\theta}-\frac{[f(\tau) \cdot f(\tau)] \theta}{2 m_{e}^{2} c^{2}}\right\} \\
& \times \sin \left(\frac{\omega^{\prime}}{\mathcal{E}(\tau)}\left\{\frac{\theta}{2}-\frac{[f(\tau) \cdot f(\tau)] \theta^{3}}{24 m_{e}^{2} c^{2}}\right\}\right) d \theta .
\end{aligned}
$$

The integral can be expressed in terms of the MacDonald function (= the modified Bessel function):

$$
\frac{d I_{\mathrm{cl}}(\tau)}{d \omega^{\prime}}=\frac{I_{\mathrm{cl}}(\tau)}{\omega_{c}} Q_{\mathrm{cl}}\left(r_{0}\right), \quad \frac{d I_{\mathrm{cl}}(\tau)}{d r_{0}}=I_{\mathrm{cl}}(\tau) Q_{\mathrm{cl}}\left(r_{0}\right),
$$

where $Q_{\mathrm{cl}}\left(r_{0}\right)$ is the unity-normalized spectrum of the gyrosynchrotron emission $\left[\int Q_{\mathrm{cl}}(r) d r=1\right]$ :

$$
Q_{\mathrm{cl}}\left(r_{0}\right)=\frac{9 \sqrt{3}}{8 \pi} r_{0} \int_{r_{0}}^{\infty} K_{5 / 3}\left(r^{\prime}\right) d r^{\prime}, \quad r_{0}=\frac{\omega^{\prime}}{\omega_{c}}
$$

and

$$
\omega_{c}=\frac{3}{2} \frac{\mathcal{E}(\tau) \sqrt{-[f(\tau) \cdot f(\tau)]}}{m_{e} c}=\frac{3}{2} \mathcal{E}(\tau) \sqrt{\frac{3 I_{\mathrm{cl}}(\tau) c}{2 e^{2}}},
$$

or, which is the same,

$$
\frac{\hbar \omega_{c}}{m_{e} c^{2}}=\mathcal{E} \sqrt{\frac{I_{\mathrm{cl}}(\tau)}{I_{C}}}=\mathcal{E} \chi .
$$

Note that despite all approximations, the integral over the frequency spectrum is consistently equal to $I_{\mathrm{cl}}$.

\section{E. Implications for strong laser fields}

As discussed, the condition $\mathcal{E} \gg 1$ and the inequality [Eq. (15)] are both fulfilled for an ultrarelativistic electron gyrating in a uniform steady-state magnetic field. By expressing the four-force squared, $-(f \cdot f)=e^{2} \mathbf{p}_{\perp}^{2} \mathbf{B}^{2}$ [see Eq. (2)], and taking the gyrofrequency in the comoving frame, $\omega_{0}^{2}$ $=\mathcal{E}^{2} e^{2} \mathbf{B}^{2} /\left(m_{e}^{2} c^{2} \mathcal{E}^{2}\right)=e^{2} \mathbf{B}^{2} /\left(m_{e}^{2} c^{2}\right)$, one finds that Eq. (15) is fulfilled as long as $\mathbf{p}_{\perp}^{2} \gg 1$.

Furthermore, application to the 1D wave field is no less straight forward. The laser wave frequency in the comoving frame, $\omega_{0}=c(k \cdot p)$, is present on the left-hand side of Eq. (5). The Lorentz four-force squared is given in Eq. (8), resulting in the following estimate for the formation time:

$$
\theta_{\mathrm{f}}=\frac{2}{c(k \cdot p)|d \mathbf{a} / d \xi|} .
$$

Now it is easy to see that the condition $\theta_{\mathrm{f}} \omega_{0} \ll 1$ as in Eq. (15) is fulfilled in relativistically strong wave field at

$$
\left|\frac{d \mathbf{a}}{d \xi}\right| \gg 1 \text {. }
$$

The formation time tends to zero as the wave amplitude tends to infinity. The change in the electron energy within the formation time is always much less than the particle energy, $\mathcal{E} m_{e} c^{2}$. Within the classical field theory this statement follows from Eqs. (8) and (19). With an account of an extra factor of $\mathcal{E}$, which arises while transforming the formation time to the laboratory frame of reference, the relative change in energy equals 


$$
\frac{\theta_{\mathrm{f}} I_{\mathrm{cl}}}{m_{e} c^{2}}=2 \alpha \chi
$$

where

$$
\alpha=\frac{e^{2}}{\hbar c} \approx \frac{1}{137}
$$

is fine structure constant. The ratio [Eq. (21)] is much less than unity as long as $\chi \leq 1$. Note that in the opposite limiting case of QED-strong field, the extra factor of $I_{\mathrm{QED}} / I_{\mathrm{cl}} \propto \chi^{-4 / 3}$ (see Fig. 3) makes ratio [Eq. (21)] small at $\chi \gg 1$ as well.

The same estimate of the formation time is applicable to any relativistically strong electromagnetic field, not only to 1D wave. Particularly, in the wakefield acceleration scheme, where the electric field in the wakefield of the pulse may be even larger than the relativistically strong field in the pulse itself. With this account, the acceleration of almost monoenergetic electron beam by the laser pulse must be accompanied by the gyrosynchrotronlike spectrum of emission (which are actually observed-see $[18,19]$ ). These observations demonstrate the general character of the gyrosynchrotron emission spectrum (this point of view, presented in [18], may be also found in Sec. 77 in [4]).

\section{F. Emission within short time intervals and implications for numerical simulation}

In strong fields satisfying the condition as in Eq. (15) both emission vector amplitude and the emission spectrum may be determined with respect to brief time interval, $\Delta t$, which may be much shorter than the field period. The only requirement is that this interval should be large as compared to the formation time:

$$
\int_{t}^{t+\Delta t} \frac{d t^{\prime}}{\mathcal{E}\left(t^{\prime}\right)} \gg \theta_{\mathrm{f}},
$$

however, the change in the field and particle characteristics within this time interval may be small, as long as $\Delta t \omega \ll 1$. In this case the span in the integral determining the vector amplitude may be chosen to be $(t, t+\Delta t)$. However, in the integral over $d \theta$, which determines the emission spectrum, the integration limits are much larger than the formation time, therefore, they may be again set to $(-\infty,+\infty)$.

These considerations justify the numerical scheme for collecting the high-frequency emission as described in [14] (which does not seem different from that briefly described in [18]). In addition to calculating the electromagnetic fields on the grid using particle-in-cell (PIC) scheme, in which fields are created by a moving particle within the time step, $\Delta t$, one can also account for the higher-frequency (subgrid) emission spectrum by calculating the instantly radiated energy, $I_{\mathrm{cl}} \Delta t$, and its distribution over frequency, parametrized via $I_{\mathrm{cl}}$. Another often used approach based on the calculation of the vector amplitude of emission (see, e.g., [20]) seems to be less efficient, although, theoretically, should provide the same result. The vector amplitude formalism, on the other hand, may be better applicable to the cases, where the high-frequency emission from multiple electrons is coherent (see [21]).
Stemming from these considerations it is now easy to proceed to the QED approach.

\section{ELECTRON IN QED-STRONG FIELD: THE EMISSION PROBABILITY}

The emission probability in the QED-strong 1D wave field may be found in Secs. 40, 90, and 101 in [9], as well as in $[16,22]$. In application to the wakefield acceleration of electrons of energy $\approx 1 \mathrm{TeV}$ the QED effects had been also discussed in [23]. However, to simulate highly dynamical effects in pulsed fields, one needs a reformulated emission probability, related to short time intervals $[$ not $(-\infty,+\infty)]$.

Indeed, it is demonstrated above that in strong fields the emission processes are essentially local functions of the instantaneous parameters. Therefore, in QED-strong fields the emission probability should be formulated in terms of the local values of the electromagnetic field intensities or, the way we adopt, it may be parametrized via the classical radiation loss rate or the Lorentz four-force squared: $-f^{\mu} f_{\mu}$ $\propto I_{\mathrm{cl}}$. This emission probability is rederived here with careful attention to consistent problem formulation and neglecting technical details.

\section{A. QED solution of the Dirac equation}

The Dirac equation which determines the evolution of the wave function, $\psi$, for a nonemitting electron in the external field reads as

$$
\left[i \chi_{C}\left(\gamma \cdot \frac{\partial}{\partial x}\right)-(\gamma \cdot a)\right] \psi=\psi,
$$

with $\gamma^{\mu}$ being the Dirac $4 \times 4$ matrices, $\left(\gamma^{0}, \gamma^{1}, \gamma^{2}, \gamma^{3}\right)$. The relativistic dot product of the Dirac matrices by four-vectors, such as $(\gamma \cdot a)$, is the linear combination of the Dirac matrices: $(\gamma \cdot a)=\gamma^{0} a^{0}-\gamma^{1} a^{1}-\gamma^{2} a^{2}-\gamma^{3} a^{3}$. Such linear combination, which is also a $4 \times 4$ matrix, may be multiplied by another matrix of this kind or by four-component bispinor, such as $\psi$, following matrix multiplication rules. For example, $(\gamma \cdot a) \psi$ is a bispinor, as is the matrix, $(\gamma \cdot a)$ multiplied from the right hand side by the bispinor, $\psi$.

By expanding Eq. (23) in the TST it is easy to find its solution in a form of a plane electron wave (the normalization coefficient $N=$ const):

$$
\psi=\frac{u[p(\xi)] P(\xi)}{\sqrt{N}} \exp \left\{\frac{i\left[\left(\mathbf{p}_{\perp 0} \cdot \mathbf{x}_{\perp}\right)-\frac{\lambda(k \cdot p) x^{1}}{\sqrt{2}}\right]}{\chi_{C}}\right\} .
$$

Here $u[p(\xi)]$ is plane wave bispinor amplitude, which satisfies the system of four linear algebraic equations:

$$
[\gamma \cdot p(\xi)] u[p(\xi)]=u[p(\xi)],
$$

as well as the normalization condition: $\hat{u} u=2$. The $\xi$-dependent momentum, $p(\xi)$, in the bispinor amplitude should be taken in accordance with Eq. (10) as for the classical trajectory of the electron. The $\xi$-dependent phase multiplier, $P(\xi)$ is as follows: 


$$
P(\xi)=\exp \left(-\frac{i}{\chi_{C}} \int^{\xi} \frac{1+\mathbf{p}_{\perp}^{2}\left(\xi_{2}\right)}{2(k \cdot p)} d \xi_{2}\right)
$$

or

$$
P(\xi)=P\left(\xi^{\prime}\right) \exp \left(-\frac{i}{\chi_{C}} \int_{\xi^{\prime}}^{\xi} \frac{1+\mathbf{p}_{\perp}^{2}\left(\xi_{2}\right)}{2(k \cdot p)} d \xi_{2}\right) .
$$

Using Eq. (10), one can find

$$
u[p(\xi)]=\left(1+\frac{(\gamma \cdot k)\left\{\gamma \cdot\left[a(\xi)-a\left(\xi^{\prime}\right)\right]\right\}}{2(k \cdot p)}\right) u\left[p\left(\xi^{\prime}\right)\right]
$$

and verify that Eq. (24) satisfies the Dirac equation. The advantage of the approach used here as compared to the known Volkov solution presented in Sec. 40 in [9] is that the wave function in Eqs. (24)-(27) is described in a selfcontained manner within some finite time interval, $\left(\xi^{\prime}, \xi\right)$ (in fact, this interval is assumed to be very short below) in terms of the local parameters of the classical trajectory of electrons. This approach is better applicable to strong fields, in which the time interval between subsequent emission occurrences, which destroys the unperturbed wave function, becomes very short.

\section{B. Matrix element for emission}

The emission problem is formulated in the following way. The electron motion in the strong field may be thought of as the sequence of short intervals. Within each of these intervals the electron follows a piece of a classical trajectory, as in Eq. (10), and its wave function (an electron state) is given by Eq. (24). The transition from one piece of the classical trajectory to another or the same from one electron state to another occurs in a probabilistic manner. The probability of this transition, which is accompanied by a photon emission is calculated below using the QED perturbation theory.

The only difficulty specific to strong pulsed fields is that the short piece of the electron trajectory is strictly bounded in space and in time, while the QED invariant perturbation theory is based on the "matrix element," which is the integral over infinite four-volume.

To avoid this difficulty the following method is suggested, which is analogous to the dipole emission theory as applied in TST. Introduce domain, $\Delta^{4} x=\left(\Delta x^{1} S_{\perp}\right) \Delta x^{0}$, bounded by two hypersurfaces, $\xi=\xi_{-}$and $\xi=\xi_{+}$(see Fig. 1). The difference $\xi_{+}-\xi_{-}$is bounded as described below so that $\Delta^{4} x$ covers only a minor part of the pulse. A volume,

$$
V=S_{\perp} \chi\left(\xi_{+}-\xi_{-}\right)=S_{\perp} \chi \int_{\xi_{-}}^{\xi_{+}} d \xi_{2},
$$

is a section of $\Delta^{4} x$ subtended by a line $t=$ const.

With the following choice for the normalization coefficient in Eq. (24):

$$
N=2 S_{\perp} \nsucc \int_{\xi_{-}}^{\xi_{+}} \mathcal{E}\left(\xi_{2}\right) d \xi_{2}
$$

the integral of the electron density in the volume $V$,

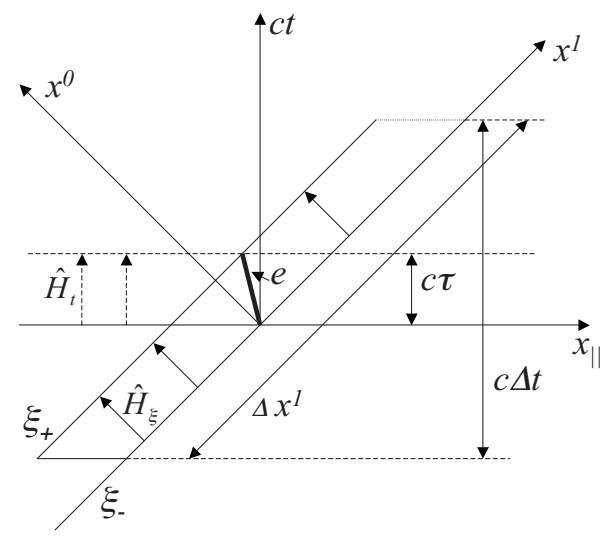

FIG. 1. The volume over which to integrate the matrix element while finding the emission probability: in the standard scheme for the dipole emission (in dashed lines) and in the TST (in solid lines). Arrows show the direction, along which the Heisenberg operator advances the wave functions.

$$
\int \hat{\psi} \gamma^{0} \psi d V=S_{\perp} \chi \int_{\xi_{-}}^{\xi_{+}} \hat{\psi} \gamma^{0} \psi d \xi_{2}
$$

is set to unity, i.e., there is a single electron in the volume $V$. This statement follows from Eq. (24) and the known property of normalized bispinor amplitudes: $\hat{u} \cdot \gamma^{0} \cdot u=2 \mathcal{E}$. Here the hat means the Dirac conjugation.

For a photon of wave vector, $\left(k^{\prime}\right)^{\mu}$, and polarization vector, $l^{\mu}$, introduce the wave function:

$$
\left(A^{\prime}\right)^{\mu}=\frac{\exp \left[-i\left(k^{\prime} \cdot x\right) / \chi_{C}\right]}{\sqrt{N_{p}}} l^{\mu}
$$

or by expanding this in the TST:

$$
\left(A^{\prime}\right)^{\mu}=\frac{P_{p}(\xi)}{\sqrt{N_{p}}} \exp \left[\frac{i\left(\mathbf{k}_{\perp}^{\prime} \cdot \mathbf{x}_{\perp}\right)}{\chi_{C}}-\frac{i \chi\left(k \cdot k^{\prime}\right) x^{1}}{\sqrt{2} \chi_{C}}\right] l^{\mu},
$$

where

$$
P_{p}(\xi)=\exp \left[-i \xi \frac{\left(\mathbf{k}_{\perp}^{\prime}\right)^{2}}{2\left(k \cdot k^{\prime}\right) \chi_{C}}\right]
$$

Here the photon momentum and photon energy are related to $m_{e} c$ and $m_{e} c^{2}$ correspondingly, or, equivalently, dimensionless $\left(k^{\prime}\right)^{\mu}$ equals dimensional $\left(k^{\prime}\right)^{\mu}$ multiplied by $\chi_{C}$. The choice of the normalization coefficient,

$$
N_{p}=\frac{\omega^{\prime} V}{2 \pi \hbar c \chi_{C}}
$$

corresponds to a single photon in the volume, $V$.

The emission probability, $d W$, is given by an integral over $\Delta^{4} x$ :

$$
d W=\frac{\alpha L_{f} L_{p}}{\hbar c}\left|\int \hat{\psi}_{f}\left[\gamma \cdot\left(A^{\prime}\right)^{*}\right] \psi_{i} d x^{0} d x^{1} d x^{2} d x^{3}\right|^{2} .
$$

Here 


$$
L_{p}=\frac{V d^{3} \mathbf{k}^{\prime}}{\left(2 \pi \chi_{C}\right)^{3}}=\frac{\hbar c N_{p} d^{2} \mathbf{k}_{\perp}^{\prime} d\left(k \cdot k^{\prime}\right)}{\left(2 \pi \chi_{C}\right)^{2}\left(k \cdot k^{\prime}\right)}
$$

is the number of states for the emitted photon. The transformation of the phase volume as in Eq. (29) is based on the following Jacobian:

$$
\left(\frac{\partial k_{\|}^{\prime}}{\partial\left(k^{\prime} \cdot k\right)}\right)_{\mathbf{k}_{\perp}^{\prime}=\text { const }}=\frac{\omega^{\prime}}{\left(k^{\prime} \cdot k\right)},
$$

which is also used below in many places. A subscript $i, f$ denotes the electron in the initial $(i)$ or final $(f)$ state. The number of electron states in the presence of the wave field, $L_{i, f}$, should be integrated over the volume $V$

$$
L_{i, f}=\frac{1}{\left(2 \pi \chi_{C}\right)^{3}} \int_{V} d^{3} \mathbf{p}_{i, f} d V=\frac{d(k \cdot p)_{i, f} d^{2} \mathbf{p}_{\perp i, f} N_{i, f}}{2(2 \pi)^{3} \chi_{C}^{3}(k \cdot p)_{i, f}} .
$$

\section{Conservation laws}

The integration by $d x^{1} d x^{2} d x^{3}=c \sqrt{2} d t d^{2} \mathbf{x}_{\perp}$ results in three $\delta$ functions, expressing the conservation of totals of $\mathbf{p}_{\perp}$ and $(k \cdot p)$, for particles in initial and final states:

$$
\mathbf{p}_{\perp i}=\mathbf{p}_{\perp f}+\mathbf{k}_{\perp}^{\prime}, \quad\left(k \cdot p_{i}\right)=\left(k \cdot p_{f}\right)+\left(k \cdot k^{\prime}\right) .
$$

Twice integrated with respect to $d x^{1}$, the probability $d W$ is proportional to a long time interval, $\Delta t=\Delta x^{1} /(c \sqrt{2})$, if the boundary condition for the electron wave at $\xi=\xi_{-}$is maintained within that long time. On transforming the integral over $d x^{0}$ to that over $d \xi$, one can find

$$
\begin{aligned}
& \left|\int \cdots d^{4} x\right|^{2} \\
& =\left(2 \pi \chi_{C}\right)^{3} S_{\perp} c \Delta t \chi\left|\int \cdots d \xi\right|^{2} \\
& \times \delta^{2}\left(\mathbf{p}_{\perp i}-\mathbf{p}_{\perp f}-\mathbf{k}_{\perp}^{\prime}\right) \delta\left[\left(k \cdot p_{i}\right)-\left(k \cdot p_{f}\right)-\left(k \cdot k^{\prime}\right)\right] .
\end{aligned}
$$

To take the large value of $\Delta t$ seems to be the only way to calculate the integral, however, the emission probability calculated in this way relates to multiple electrons in the initial state, each of them locating between the wave fronts $\xi=\xi_{-}$ and $\xi=\xi_{+}$during much shorter time,

$$
\delta t\left(\xi_{-}, \xi_{+}\right)=(1 / c) \int_{\xi_{-}}^{\xi^{+}} \mathcal{E}_{i}\left(\xi_{2}\right) d \xi_{2} /\left(k \cdot p_{i}\right) .
$$

For a single electron the emission probability becomes

$$
d W_{f i}\left(\xi_{-}, \xi_{+}\right)=\delta t d W / \Delta t
$$

Using $\delta$ functions it is easy to integrate Eq. (28) over $d \mathbf{p}_{\perp f} d\left(k \cdot p_{f}\right)$ :

$$
\frac{d W_{f i}\left(\xi_{-}, \xi_{+}\right)}{d\left(k \cdot k^{\prime}\right) d^{2} \mathbf{k}_{\perp}^{\prime}}=\frac{\alpha\left|\int_{\xi_{-}}^{\xi_{+}} T(\xi) \hat{u}\left(p_{f}\right)\left(\gamma \cdot l^{*}\right) u\left(p_{i}\right) d \xi\right|^{2}}{\left(4 \pi \chi_{C}\right)^{2}\left(k \cdot k^{\prime}\right)\left(k \cdot p_{i}\right)\left(k \cdot p_{f}\right)},
$$

where

$$
T(\xi)=\frac{P_{i}(\xi)}{P_{f}(\xi) P_{p}(\xi)}=\exp \left[\frac{i \int^{\xi}\left(k^{\prime} \cdot p_{i}\left(\xi_{2}\right)\right) d \xi_{2}}{\chi_{C}\left(k \cdot p_{f}\right)}\right],
$$

$P_{i}(\xi)$ and $P_{f}(\xi)$ are the electron phase multipliers, $P(\xi)$, for the electron in initial and final states, and

$$
p_{f}^{\mu}(\xi)=p_{i}^{\mu}(\xi)-\left(k^{\prime}\right)^{\mu}+\frac{\left(k^{\prime} \cdot p_{i}(\xi)\right)}{\left(k \cdot p_{i}\right)-\left(k \cdot k^{\prime}\right)} k^{\mu} .
$$

Prior to discussing Eq. (31), return to Eq. (27) and analyze it component by component in the TST. It appears that three of the four components of that equation describe the conservation of $(k \cdot p)$ and $\mathbf{p}_{\perp 0}=\mathbf{p}_{\perp}+\mathbf{a}$ for electron in the course of its emission-free motion. At the same time, yet another component of Eq. (27), specifically, $p^{1}$, directed along $k^{\mu}$, describes the energy-momentum exchange between the electron and the 1D wave field, maintaining the identity, $(p \cdot p)=1$. Now turn to Eq. (31). Again, three of the four components express the conservation of the same variables in the course of the photon emission, while the $p^{1}$ component, directed along $k^{\mu}$ describes the absorption of energy and momentum from the wave field in the course of the photon emission. Note that in the case of a strong field the energy absorbed from field is not an integer number of quanta and that for short nonharmonic field it is not even a constant but a function of the local field.

\section{Calculation of the matrix element}

To calculate the matrix element, one can rewrite it as the double integral over $d \xi d \xi_{1}$ and then reduce the matrices $u\left[p_{i, f}(\xi)\right] \otimes \hat{u}\left[p_{i, f}\left(\xi_{1}\right)\right]$ in the integrand to the polarization matrices of the electron at $\xi$ or at $\xi_{1}$ using Eq. (27). These standard manipulations with the Dirac matrices are omitted here. Although in a strong wave electrons may be polarized (see [24]), in the present work the emission probability is assumed to be averaged over the electron initial polarizations and summed over its final polarizations. The ultimate result of these derivations is as follows:

$$
\frac{d W_{f i}}{d\left(k \cdot k^{\prime}\right) d^{2} \mathbf{k}_{\perp}^{\prime}}=\frac{\left.\alpha \int_{\xi_{-}^{+}}^{\xi_{+}} \int_{\xi_{-}}^{\xi_{+}} T(\xi) T\left(-\xi_{1}\right)\right] D d \xi d \xi_{1}}{\left(2 \pi \chi_{C}\right)^{2}\left(k \cdot k^{\prime}\right)\left(k \cdot p_{i}\right)\left(k \cdot p_{f}\right)},
$$

where

$$
D=\left[l^{*} \cdot p_{i}\left(\xi_{1}\right)\right]\left[l \cdot p_{i}(\xi)\right]-\frac{\left\{\left[p(\xi) \cdot p\left(\xi_{1}\right)\right]-1\right\}\left(1-C_{f i}\right)^{2}}{4 C_{f i}}
$$

and

$$
C_{f i}=\frac{\left(k \cdot p_{f}\right)}{\left(k \cdot p_{i}\right)}=1-\frac{\chi_{C}\left(k \cdot k^{\prime}\right)}{\left(k \cdot p_{i}\right)} \leq 1
$$

is a recoil parameter which characterizes the reduction in the photon momentum due to emission.

The matrix element may also be summed, if desired, for two possible directions of the polarization vector. The second term in the integrand is simply multiplied by two, while in the first one the negative of the metric tensor should be substituted for the product of the polarization vectors (see Sec. 8 in [9]) so that $-\left[p_{i}(\xi) \cdot p_{i}\left(\xi_{1}\right)\right]$ substitutes for 
$\left[l^{*} \cdot p_{i}\left(\xi_{1}\right)\right]\left[l \cdot p_{i}(\xi)\right]$. The latter may be transformed using Eq. (11), thus giving

$$
\sum_{l} D=-\left(\frac{\left\{\left[p(\xi) \cdot p\left(\xi_{1}\right)\right]-1\right\}\left(1+C_{f i}^{2}\right)}{2 C_{f i}}+1\right) .
$$

\section{E. Vector amplitude of emission in QED case}

Now moving to connection between the obtained result, on one hand, and the way the high-frequency emission is treated in the framework of classical theory, on the other. To facilitate the comparison, both here and in Sec. II the photon frequency and wave vector, $\omega^{\prime}$ and $\mathbf{k}^{\prime}$, are not dimensionless. It appears that the QED result obtained above can be reformulated in a form similar to Eq. (13). Using the following relationships between the differentials:

$$
\begin{gathered}
d t=\mathcal{E} d \tau=\frac{\mathcal{E}}{c} \frac{d \xi}{(k \cdot p)}, \quad d R_{\mathrm{QED}}=\hbar \omega^{\prime} d W_{f i}, \\
\frac{\left(\omega^{\prime}\right)^{2} d \omega^{\prime} d \mathbf{n}}{c^{3}}=d^{3} \mathbf{k}^{\prime}=\frac{\omega^{\prime} d^{2} \mathbf{k}_{\perp}^{\prime} d\left(k \cdot k^{\prime}\right)}{c\left(k \cdot k^{\prime}\right)},
\end{gathered}
$$

one can reduce Eq. (32) for the polarized part of the emission to the same form as that of Eq. (13):

$$
\frac{d R_{\mathrm{QED}}^{\mathrm{pol}}}{d \omega^{\prime} d \mathbf{n}}=\frac{\left(\omega^{\prime}\right)^{2}}{4 \pi^{2} c}\left|\left(\mathbf{A}_{\mathrm{QED}}\left(\omega^{\prime}\right) \cdot \mathbf{I}^{*}\right)\right|^{2},
$$

where

$$
\mathbf{A}_{\mathrm{QED}}\left(\omega^{\prime}\right)=\sqrt{\frac{e^{2}}{C_{f i}}} \times \int_{t_{-}}^{t_{+}} \frac{\mathbf{v}(t)}{c} \exp \left\{\frac{i \omega^{\prime}}{C_{f i}}\left[t-\frac{(\mathbf{n} \cdot \mathbf{r}(t))}{c}\right]\right\} d t,
$$

where $t_{-}, t_{+}$are the time instants when the electron crosses the hypersurfaces $\xi=\xi_{-}$and $\xi=\xi_{+}$correspondingly. In the considered strong field case the finite integration limits are admissible as long as the integration span well exceeds the formation time. Therefore,

$$
\begin{aligned}
& \mathbf{A}_{\mathrm{QED}}\left(\omega^{\prime}\right)=\sqrt{\frac{1}{C_{f i}}} \mathbf{A}_{\mathrm{cl}}\left(\frac{\omega^{\prime}}{C_{f i}}\right), \\
& \frac{d I_{\mathrm{QED}}^{\mathrm{pol}}\left(\omega^{\prime}\right)}{d \omega^{\prime} d \mathbf{n}}=C_{f i} \frac{d I_{\mathrm{cl}}\left(\omega^{\prime} / C_{f i}\right)}{d \omega^{\prime} d \mathbf{n}} .
\end{aligned}
$$

Thus, the QED effect on the emission from an electron reduces to the classical electric-dipole emission from a moving charge in an electromagnetic field with a very simple rule to transform the polarized emission intensity and polarized emission amplitude, which accounts for the recoil effect.

However, the electron in the QED-strong field emits not only as an electric charge because it also possesses a magnetic moment associated with its spin. In Eq. (32) a depolarized contribution to the emission is present. This contribution may be related to the electron spin, which is assumed to be depolarized. The depolarized emission energy related to the interval of frequency and to the element of solid angle and summed over two polarization directions (i.e., multiplied by two) equals

$$
\begin{aligned}
\frac{d R_{\mathrm{QED}}^{\mathrm{depol}}}{d \omega^{\prime} d \mathbf{n}}= & \frac{\left(\omega^{\prime}\right)^{2}}{4 \pi^{2} c} \frac{\left(1-C_{f i}\right)^{2}}{2 C_{f i}} \\
& \times\left\{\sum_{\mathbf{l}}\left|\left(\mathbf{A}_{\mathrm{QED}}\left(\omega^{\prime}\right) \cdot \mathbf{I}^{*}\right)\right|^{2}+\left|\varphi_{\mathrm{QED}}\right|^{2}\right\},
\end{aligned}
$$

where

$$
\varphi_{\mathrm{QED}}=\sqrt{\frac{e^{2}}{C_{f i}}} \int_{t_{-}}^{t_{+}} \exp \left(\frac{i \omega^{\prime}}{C_{f i}}\left\{t-\frac{[\mathbf{n} \cdot \mathbf{r}(t)]}{c}\right\}\right) \frac{d t}{\mathcal{E}(t)}
$$

is a scalar amplitude of emission introduced in a way similar to that for the vector amplitude. After derivations analogous to those of Sec. II, the radiation loss rate due to the electron magnetic moment reads as

$$
\frac{d I_{\mathrm{QED}}^{\mathrm{depol}}}{d \omega^{\prime}}=\frac{I_{\mathrm{cl}}(\tau)}{\omega_{c}} \frac{9 \sqrt{3}}{8 \pi}\left(1-C_{f i}\right)^{2} \frac{r_{0}}{C_{f i}} K_{2 / 3}\left(\frac{r_{0}}{C_{f i}}\right) .
$$

Thus, the QED effect in the emission from an electron in a strong electromagnetic field reduces to a downshift in frequency accompanied by an extra contribution from the magnetic moment of electron. Note a general character of these conclusions: only in the recoil parameter, $C_{f i}$, is there a direct dependence on the $1 \mathrm{D}$ wave vector. This dependence can be also excluded because, for the photons emitted along the direction of the particle motion, the following approximation is valid:

$$
C_{f i} \approx 1-\frac{\hbar \omega^{\prime}}{\mathcal{E} m_{e} c^{2}}=1-\chi r_{0}, \quad \frac{r_{0}}{C_{f i}}=r_{\chi}=\frac{r_{0}}{1-\chi r_{0}}
$$

so that in QED-strong fields the emission spectrum is also universal and may be parametrized with the sole parameter, $I_{\mathrm{cl}}$.

Combining Eqs. (19), (22), and (30) one can derive the condition

$$
\xi_{+}-\xi_{-} \gg \frac{2}{|d \mathbf{a} / d \xi|} .
$$

Under this condition, the time interval within which the emitting electron locates between the wave fronts $\xi=\xi_{-}$and $\xi=\xi_{+}$much exceeds the formation time of emission validating the above considerations.

With these results the scheme to account for the highfrequency emission as outlined in Sec. II may be easily extended to 3D QED-strong fields. However, the radiation back reaction needs to be incorporated for this scheme to be consistent.

\section{RADIATION AND ITS BACK-REACTION}

Unless the field is QED strong the radiation back-reaction in a relativistically strong laser wave may or may not be significant. The condition for the field to be radiationdominant (see, e.g., Ref. [8]) is formulated in terms of the ratio between the magnitudes of the Lorentz force and the radiation force, which become comparable at intensities $J$ $\sim 10^{23} \mathrm{~W} / \mathrm{cm}^{2}$. For an electron moving toward the laser wave, the radiation force starts to dominate at a lower wave 
intensity depending on the electron energy [8]. The radiation back-reaction decelerates such an electron, the effect being more pronounced for longer laser pulses [14]. As the result, at intensities $J \sim 10^{22} \mathrm{~W} / \mathrm{cm}^{2}$ the radiation back-reaction drastically changes the character of the laser pulse interaction with dense plasmas and $\gamma$-ray emission becomes a leading mechanism of the laser energy losses [25].

In QED-strong fields the radiation back-reaction is always significant, as long as at each photon emission the electron looses a noticeable fraction of its momentum and energy. The matter of principle is also a consistency of the perturbation theory of emission in QED-strong fields. Within the framework of classical theory the momentum-energy change resulting from the radiation back-reaction should be small in some sense to properly approximate the radiation force [see Refs. $[4,15]$ as well as the considerations relating to the estimate as in Eq. (21)]. In QED-strong fields this change cannot be claimed to be small, but the probability of emission can be. Specifically, the difference, $\xi_{+}-\xi_{-}$, should be small enough, so that the probability of emission within the time interval of Eq. (30) should be much less (or at least less) than unity:

$$
\int \frac{d W_{f i}}{d \mathbf{k}_{\perp}^{\prime} d\left(k^{\prime} \cdot k\right)} d \mathbf{k}_{\perp}^{\prime} d\left(k^{\prime} \cdot k\right) \ll 1 .
$$

\section{A. Emission probability and radiation loss rate}

The derivations performed in Sec. II for the radiation loss rate, namely, the approximation of the angular distribution with the Dirac function and approximation of the frequency spectrum with the MacDonald functions may be directly applied to the emission probability.

On developing the dot product, $\left(k^{\prime} \cdot p_{i}\right)$, in $T(\xi)$ in the TST metric, $G^{\mu \nu}$, one can find

$$
T(\xi) T\left(-\xi_{1}\right)=\exp \left[i\left(T_{1}+T_{2}\right)\right],
$$

where

$$
\begin{gathered}
T_{1}=\frac{\left(k \cdot p_{i}\right)}{2 \chi_{C}\left(k \cdot k^{\prime}\right)\left(k \cdot p_{f}\right)}\left(\frac{\left(k \cdot k^{\prime}\right)}{\left(k \cdot p_{i}\right)}\left\langle\mathbf{p}_{\perp i}\right\rangle-\mathbf{k}_{\perp}^{\prime}\right)^{2}\left(\xi-\xi_{1}\right), \\
T_{2}=\frac{\left(k \cdot k^{\prime}\right)\left\{\left(\xi-\xi_{1}\right)+\int_{\xi_{1}}^{\xi}\left[\mathbf{a}\left(\xi_{2}\right)-\langle\mathbf{a}\rangle\right]^{2} d \xi_{2}\right\}}{2 \chi_{C}\left(k \cdot p_{i}\right)\left(k \cdot p_{f}\right)}, \\
\langle\mathbf{a}\rangle=\frac{\int_{\xi_{1}}^{\xi} \mathbf{a} d \xi_{2}}{\xi-\xi_{1}}, \quad\left\langle\mathbf{p}_{\perp i}\right\rangle=\mathbf{p}_{\perp 0 i}-\langle\mathbf{a}\rangle .
\end{gathered}
$$

Integration over $d^{2} \mathbf{k}_{\perp}^{\prime}$ then gives

$$
\frac{d W_{f i}\left(\xi_{-}, \xi_{+}\right)}{d\left(k \cdot k^{\prime}\right)}=\frac{\alpha \int_{\xi_{-}}^{\xi_{+}} \int_{\xi_{-}}^{\xi_{+}} \frac{i \exp \left(i T_{2}\right)}{\xi-\xi_{1}} \sum_{l} D\left(\xi, \xi_{1}\right) d \xi d \xi_{1}}{2 \pi \chi_{C}\left(k \cdot p_{i}\right)^{2}} .
$$

In strong fields the following estimates may be applied:

$$
\left(k \cdot k^{\prime}\right) \sim \chi_{C}\left(k \cdot p_{i}\right)^{2}\left|\frac{d \mathbf{a}}{d \xi}\right|, \quad d W_{f i} \sim \alpha\left|\left(\xi_{+}-\xi_{-}\right) \frac{d \mathbf{a}}{d \xi}\right| .
$$

Now the bounds for $\xi_{+}-\xi_{-}$can be consistently introduced:

$$
|d \mathbf{a} / d \xi|^{-1} \ll \xi_{+}-\xi_{-} \ll \min \left(\alpha^{-1}|d \mathbf{a} / d \xi|^{-1}, 1\right) .
$$

Under these bounds, first, condition (35) is satisfied. Therefore, the time interval [Eq. (30)] is much greater than the formation time and the emission probability is linear in $\xi_{+}-\xi_{-}$:

$$
d W_{f i}\left(\xi_{-}, \xi_{+}\right)=(d W / d \xi)\left(\xi_{+}-\xi_{-}\right) .
$$

Second, the emission probability satisfies condition (36). Therefore, perturbation theory is applicable. In addition, the emission probability can be expressed in terms of the local electric field. Note that consistency in Eq. (37) is ensured in relativistically strong electromagnetic fields as long as $\alpha$ $\ll 1$, with no restriction on the magnitude of the electromagnetic field experienced by an electron.

Under condition (37) the probability may be expressed in terms of MacDonald functions:

$$
\begin{gathered}
\frac{d W_{f i}}{d r_{0} d \xi}=\frac{\alpha \chi\left[\int_{r_{\chi}}^{\infty} K_{5 / 3}(y) d y+r_{0} r_{\chi} \chi^{2} K_{2 / 3}\left(r_{\chi}\right)\right]}{\sqrt{3} \pi \chi_{C}\left(k \cdot p_{i}\right)}, \\
r_{0}=\frac{\left(k \cdot k^{\prime}\right)}{\chi\left(k \cdot p_{i}\right)}, \quad \chi=\frac{3}{2}\left(k \cdot p_{i}\right)\left|\frac{d \mathbf{a}}{d \xi}\right| \chi_{C}=\sqrt{\frac{I_{\mathrm{cl}}}{I_{C}}} .
\end{gathered}
$$

Probability (similar to that found in [16]) is expressed in terms of functions of $r_{0}$ and related to interval of $d r_{0}$. The way to introduce $r_{0}$ and $\chi$ looks different from that adopted in Eqs. (17) and (18), however, the difference is negligible as long as $\omega^{\prime} / \mathcal{E}_{i} \approx\left(k \cdot k^{\prime}\right) /\left(k \cdot p_{i}\right)$ for collinear $k^{\prime}$ and $p_{i}$. The momentum of the emitted radiation, related to the interval of the electron proper time, may be found from Eqs. (30) and (38):

$$
\begin{aligned}
\frac{d p_{\mathrm{rad}}}{d \tau} & =\int k^{\prime} \frac{c\left(k \cdot p_{i}\right) d W_{f i}}{d\left(k \cdot k^{\prime}\right) d^{2} \mathbf{k}_{\perp} d \xi} d\left(k \cdot k^{\prime}\right) d^{2} \mathbf{k}_{\perp} \\
& =\left\{p_{i}+k O\left[\left(k \cdot p_{i}\right)^{-1}\right]\right\} \int c\left(k^{\prime} \cdot k\right) \frac{d W}{d r_{0} d \xi} d r_{0} .
\end{aligned}
$$

As with other four-momenta, $p_{\text {rad }}$ is related to $m_{e} c$. To prove the four-vector relationship [Eq. (39)], its components in the TST metric should be integrated over $\mathbf{k}_{\perp}$ using the symmetry of $T_{1}$. The small term, $O\left[1 /\left(k \cdot p_{i}\right)\right]$, arises from the electron rest mass energy and from the small $\left(\sim 1 /\left|p_{\perp}\right|\right)$ but finite width of the photon angular distribution. In neglecting this term:

$$
\frac{d p_{\mathrm{rad}}}{d \tau}=p_{i} \frac{1}{m_{e} c^{2}} \int \frac{d I_{\mathrm{QED}}}{d r_{0}} d r_{0},
$$

$$
I_{\mathrm{QED}}=m_{e} c^{2} \int c\left(k \cdot k^{\prime}\right) \frac{d W_{f i}}{d \xi d r_{0}} d r_{0}
$$

being the radiation loss rate. The photon energy spectrum, $d I_{\mathrm{OED}} / d r_{0}$, is described as a function only of the random scalar, $r_{0}$, using only the parameter, $\chi$ (see Fig. 2). The latter may be parametrized in terms of the radiation loss rate evaluated within the framework of classical theory [see Eq. (7) and Fig. 3]. The expressions for $q\left(I_{\mathrm{cl}}\right)=I_{\mathrm{QED}} / I_{\mathrm{cl}}$ and for the normalized spectrum function, $Q\left(r_{0}, \chi\right)$, coincide with for- 


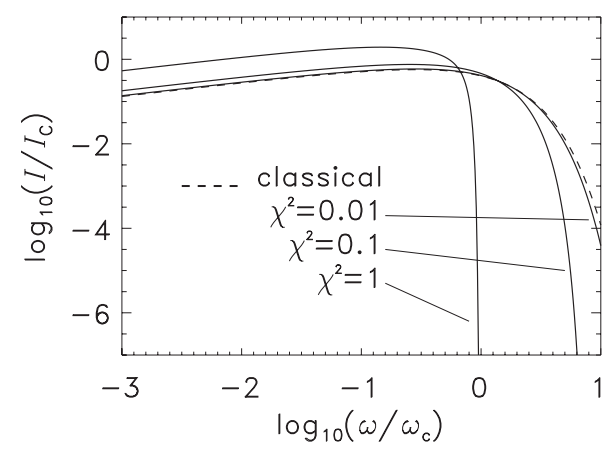

FIG. 2. Emission spectra for various values of $\chi$.

mulas known from the gyrosynchrotron emission theory (see Sec. 90 in [9]):

$$
\begin{aligned}
& q=\frac{9 \sqrt{3}}{8 \pi} \int_{0}^{\infty} d r_{0} r_{0}\left[\int_{r_{\chi}}^{\infty} K_{5 / 3}(y) d y+r_{0} r_{\chi} \chi^{2} K_{2 / 3}\left(r_{\chi}\right)\right], \\
& Q\left(r_{0}, \chi\right)=\frac{9 \sqrt{3} r_{0}}{8 \pi q}\left[\int_{r_{\chi}}^{\infty} K_{5 / 3}(y) d y+r_{0} r_{\chi} \chi^{2} K_{2 / 3}\left(r_{\chi}\right)\right] .
\end{aligned}
$$

As mentioned above,

$$
\frac{d I_{\mathrm{QED}}}{d r_{0}}=\frac{d I_{\mathrm{QED}}^{\text {pol }}}{d r_{0}}+\frac{d I_{\mathrm{QED}}^{\text {depol }}}{d r_{0}},
$$

where polarized and nonpolarized contributions are given by Eqs. (33) and (34).

\section{B. Radiation back-reaction: Radiation force approximation}

While emitting a photon, an electron also acquires fourmomenta from the external field [see Eq. (31)]:

$$
d p_{F}^{\mu}=\frac{\left(k^{\prime} \cdot p_{i}(\xi)\right)}{\left(k \cdot p_{i}\right)-\left(k \cdot k^{\prime}\right)} k^{\mu} .
$$

The interaction with the field ensures that the total effect of emission on the electron not to break the entity $\left(p_{f} \cdot p_{f}\right)=1$. As long as the angular distribution of emission is approximated with the Dirac function, the expression for $d p_{F}^{\mu}$ needs to be corrected to ensure exact momentum-energy conserva-

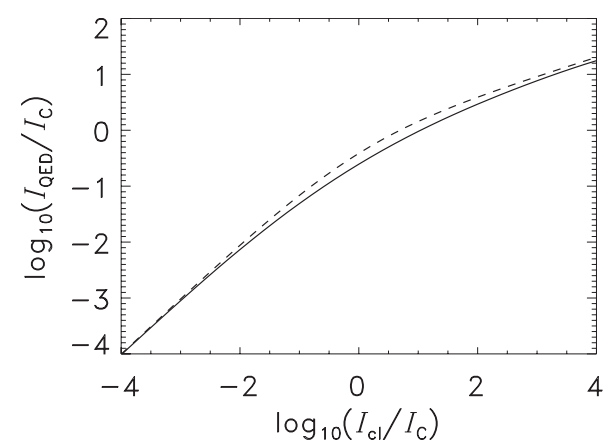

FIG. 3. Emitted radiation power in the QED approach $v s$ classical (solid); an interpolation formula $I_{\mathrm{QED}}=I_{\mathrm{cl}} /\left(1+1.04 \sqrt{I_{\mathrm{cl}} / I_{C}}\right)^{4 / 3}$ (dashed). tion with approximated momentum of radiation. The choices of near-unity correction coefficients in $d p_{F}$ are somewhat different in the cases $\chi \leq 1$ and $\chi \gg 1$.

For moderate values of $\chi \leq 1$ the radiation force $\left(d p_{F}-d p_{\text {rad }}\right) / d \tau$ may be introduced. In this approximation it is admitted that the change in the electron momentum within the infinitesimal time interval is also infinitesimal. This "Newton's law" approximation is pertinent to classical physics and it both ignores the point that the change in the electron momentum at $\chi \sim 1$ is essentially finite because of the finite momentum of emitted photon and breaks the low bound on the time interval presented in Eq. (35). The approximation, however, is highly efficient and allows one to avoid time-consuming statistical simulations. The approximation error tends to zero as $\chi \rightarrow 0$, however, it is not huge at $\chi \sim 1$ and even at $\chi=10$. The latter can be seen from Fig. 5 given below in which the average relative change in the electron energy in the course of single-photon emission is presented (assumed to be negligible within the radiation force approximation).

Within the radiation force approximation the best correction is $d p_{F}^{\mu} \approx k^{\mu}\left(k^{\prime} \cdot p_{i}\right) /\left(k \cdot p_{i}\right)$. The total radiation force may now be found by integrating $d p_{F}$ over $d\left(k^{\prime} \cdot k\right)$ :

$$
\frac{d\left(p_{f}^{\mu}-p_{i}^{\mu}\right)}{d \tau}=\left(k^{\mu} \frac{\left(p_{i} \cdot p_{i}\right)}{\left(k \cdot p_{i}\right)}-p_{i}^{\mu}\right) \frac{I_{\mathrm{QED}}}{m_{e} c^{2}} .
$$

The radiation force maintains the abovementioned entity as long as $\left[p_{i} \cdot d\left(p_{f}-p_{i}\right) / d \tau\right]=0$. Equation (40) is presented in a form which is applicable both with dimensionless or with dimensional momenta.

In $[13,14]$ it was mentioned that QED is not compatible with the traditional approach to the radiation force in classical electrodynamics and an alternative equation of motion for a radiating electron was suggested:

$$
\frac{d p^{\mu}}{d \tau}=\Omega^{\mu \nu} p_{\nu}-\frac{I_{\mathrm{QED}}}{m_{e} c^{2}} p^{\mu}+\tau_{0} \frac{I_{\mathrm{QED}}}{I_{\mathrm{cl}}} \Omega^{\mu \nu} \Omega_{\nu \beta} p^{\beta},
$$

where $\Omega^{\mu \nu}=e F^{\mu \nu} /\left(m_{e} c\right), F^{\mu \nu}$ is the field tensor, and

$$
\tau_{0}=2 e^{2} /\left(3 m_{e} c^{3}\right)=(2 / 3) \alpha \chi_{C} / c .
$$

In the 1D plane wave the particular expression for the radiation force can be found using the following equation:

$$
\tau_{0} \Omega^{\mu \nu} \Omega_{\nu \beta} p^{\beta}=k^{\mu} \frac{(p \cdot p) I_{\mathrm{cl}}}{m_{e} c^{2}(k \cdot p)} .
$$

With this account, the radiation force in Eq. (41) is the same as its QED formulation in Eq. (40). This proves that the earlier derived Eq. (41) has a wide range of applicability including electron quasiclassical motion in QED-strong fields and in the particular case of 1D wave fields it can be directly derived from the QED principles. Note that the efforts to derive the radiation force from quantum mechanics were applied many times [see [26], the most convincing approach which gives the equation quite similar to Eq. (41) may be found in [27]]. However, for the first time the derivation from QED side is provided, with the resulting equation being different from those given in textbooks $[4,15]$. 


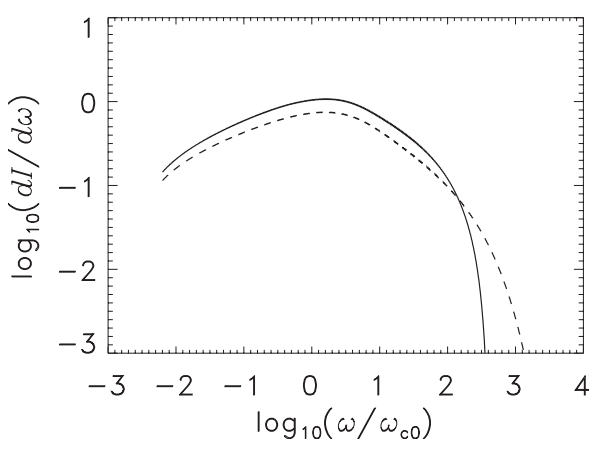

FIG. 4. The emission spectrum for $600 \mathrm{MeV}$ electrons interacting with $30 \mathrm{fs}$ laser pulses of intensity $2 \times 10^{22} \mathrm{~W} / \mathrm{cm}^{2}$ : with (solid) or without (dashed) accounting for the QED effects. Here $\hbar \omega_{c 0} \approx 1.1 \mathrm{MeV}$ for $\lambda=0.8 \mu \mathrm{m}$.

The way to solve Eq. (41) within the PIC scheme and integrate the emission is described in [14]. In Fig. 4 we show the numerical result for an electron interacting with a laser pulse. We see that the QED effects essentially modify the radiation spectrum even with laser intensities which are already achieved.

\section{Radiation back-reaction: Monte Carlo approach}

The radiation force approximation does not fully account for the statistical character of the emission process at $\chi \geq 1$. Specifically, we mentioned above that in the Newton's law approximation, the force, $\mathbf{f}$, provides only the infinitesimal change in the electron momentum, $\Delta \mathbf{p}=\mathbf{f} \Delta t \rightarrow 0$ over an infinitely short time interval, as $\Delta t \rightarrow 0$. For radiation processes in QED-strong fields this point is in contradiction with a small probability, $\Delta t \cdot d W_{f i} / d t \rightarrow 0$, for an electron to acquire a finite change in momentum, $|\delta \mathbf{p}| \sim|\mathbf{p}|$, in the course of emission.

A more quantitative, though more cumbersome, description may be achieved within the QED Monte Carlo approach. It is convenient to relate the emission probability to an interval of proper time, $\Delta \tau=\Delta t / \mathcal{E}_{i}$. From (38) it follows that

$$
\frac{d W_{f i}}{d r_{0} d \tau}=\frac{I_{\mathrm{QED}}}{m_{e} c^{2}} \frac{Q\left(r_{0}, \chi\right)}{\chi r_{0}} .
$$

[Note that on multiplying Eq. (42) by $m_{e} c^{2}\left(\omega^{\prime} / \mathcal{E}_{i}\right)$ $\approx m_{e} c^{2} \chi r_{0}$, one obtains again the formula for spectral distribution of energy emitted per interval of time.] As long as Eq. (42) for the differential probability is available, one can find the expected energy of the emitted photon:

$$
\frac{1}{\mathcal{E}_{i}}\left\langle\omega^{\prime}\right\rangle=\frac{\chi}{\int_{0}^{1 / \chi} Q\left(r_{0}, \chi\right)\left(d r_{0} / r_{0}\right)} .
$$

A plot of the expectation for the ratio $\left\langle\omega^{\prime} / \mathcal{E}_{i}\right\rangle$ vs $\chi$ is given in Fig. 5, with energy of emitted photons being denoted as $\Delta \mathcal{E}$. The total probability of emission within the interval of proper time is given by a complete integral of probability:

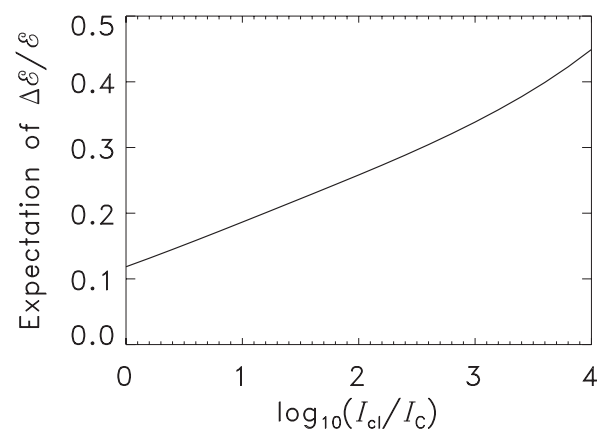

FIG. 5. Expectation of the emitted photon energy. In dimensional units $\Delta \mathcal{E}=\hbar \omega^{\prime}$ and $\mathcal{E}$ is the dimensional energy of an electron prior to emission.

$$
W_{f i}=\Delta \tau \int \frac{d W_{f i}}{d r_{0} d \tau} d r_{0}=\Delta \tau \frac{I_{\mathrm{QED}}}{m_{e} c^{2}}\left\langle\frac{\omega^{\prime}}{\mathcal{E}_{i}}\right\rangle^{-1}
$$

Both within the QED perturbation theory and within the Monte Carlo scheme $W$ is assumed to be small $W<1$. The probability of no emission equals $1-W \geq 0$. The partial probability, $W_{f i}\left(\omega^{\prime}<\omega_{0}^{\prime}\right)$, for the emission with the photon energy not exceeding the given value, $\omega_{0}^{\prime}$, is given by the incomplete probability integral:

$$
W_{f i}\left(\omega^{\prime}<\omega_{0}^{\prime}\right)=W_{f i}\left\langle\frac{\omega^{\prime}}{\mathcal{E}_{i}}\right\rangle \int^{\omega^{\prime} /\left(\mathcal{E}_{i} \chi\right)} Q\left(r_{0}, \chi\right) \frac{d r_{0}}{\chi r_{0}} .
$$

Therefore, for a given interval of proper time and calculated $\chi,\left\langle\omega^{\prime}\right\rangle / \mathcal{E}_{i}$, and $W_{f i}<1$, the expression of the only scalar to gamble, $\omega^{\prime} / \mathcal{E}_{i}$, in terms of a random number, $0 \leq \mathrm{rnd}<1$, is implicitly given by an integral equation as follows:

$$
\int_{0}^{\omega_{0}^{\prime} /\left(\mathcal{E}_{i} \chi\right)} Q\left(r_{0}, \chi\right) \frac{d r_{0}}{\chi r_{0}}=\frac{\mathrm{rnd}}{W_{f i}}\left\langle\frac{\omega^{\prime}}{\mathcal{E}_{i}}\right\rangle^{-1},
$$

if the gambled value of rnd does not exceed $W_{f i}: 0 \leq$ rnd $\leq W_{f i}$. Otherwise, i.e., if $W_{f i}<\mathrm{rnd} \leq 1$, the emission within this interval does not occur.

Once the value of $\omega^{\prime} / \mathcal{E}_{i}$ is found, the change in the electron four-momentum due to single photon emission during the time interval, $\Delta \tau$, may be determined as follows:

$$
p_{f}^{\mu}-p_{i}^{\mu}=\left\{k^{\mu} \frac{\left(p_{i} \cdot p_{i}\right)\left[1-\omega^{\prime} /\left(2 \mathcal{E}_{i}\right)\right]}{\left(k \cdot p_{i}\right)\left[1-\omega^{\prime} / \mathcal{E}_{i}\right]}-p_{i}^{\mu}\right\} \frac{\omega^{\prime}}{\mathcal{E}_{i}} .
$$

It is easy to see that the identity $\left(p_{f} \cdot p_{f}\right)=1$ is maintained. To achieve this, a correction factor, $1-\omega^{\prime} /\left(2 \mathcal{E}_{i}\right)$ is applied to the momentum exchange with the wave field as present in Eq. (31).

The implementation of this method for $3 \mathrm{D}$ realistic laser fields together with simulation results and an account for pair production will be described in detail in a forthcoming publication. 


\section{CONCLUSION}

QED-strong fields in the focus of an ultrabright laser may be realized, if desired, using the technologies which already exist. In any case, these effects will come into power when laser-plasma interactions are explored with the next generation of lasers.

It is demonstrated that electron motion in very strong laser fields with pronounced QED effects may be successfully described within the radiation force approximation. The necessary corrections in the radiation force and the emission spectra to account for the QED effects are parametrized by the sole parameter, $I_{\mathrm{cl}}$.

\section{ACKNOWLEDGMENTS}

We acknowledge an invaluable help and fruitful advices we received from S. S. Bulanov. V. T. Tikhonchuk kindly pointed out some effects we missed. We are grateful to J. Rafelski, R. F. O'Connel, and V. Hnizdo for critical comments and to V. Tenishev for discussing the Monte Carlo method. The work of one of the authors (I.S.) on high energy density physics was supported by the DOE NNSA under the Predictive Science Academic Alliances Program by Grant No. DE-FC52-08NA28616.
[1] J. Schwinger, Phys. Rev. 82, 664 (1951); E. Brezin and C. Itzykson, Phys. Rev. D 2, 1191 (1970).

[2] M. Marklund and P. K. Shukla, Rev. Mod. Phys. 78, 591 (2006); Y. I. Salamin et al., Phys. Rep. 427, 41 (2006).

[3] A. R. Bell and J. G. Kirk, Phys. Rev. Lett. 101, 200403 (2008).

[4] L. D. Landau and E. M. Lifshits, The Classical Theory of Fields (Pergamon, New York, 1994); The Classical Theory of Fields, 1st ed. (Moscow, Gostekhizdat, 1941).

[5] A. Di Piazza, K. Z. Hatsagortsyan, and C. H. Keitel, Phys. Rev. Lett. 97, 083603 (2006); S. S. Bulanov, Phys. Rev. E 69, 036408 (2004).

[6] S.-W. Bahk et al., Opt. Lett. 29, 2837 (2004); V. Yanovsky et al., Opt. Express 16, 2109 (2008).

[7] http://www.extreme-light-infrastructure.eu/; E. Gerstner, Nature (London) 446, 16 (2007).

[8] J. Koga, T. Zh. Esirkepov, and S. V. Bulanov, Phys. Plasmas 12, 093106 (2005).

[9] V. B. Berestetskii, E. M. Lifshitz, and L. P. Pitaevskii, Quantum Electrodynamics (Pergamon, Oxford, 1982).

[10] C. Bula et al., Phys. Rev. Lett. 76, 3116 (1996); D. L. Burke, R. C. Field, G. Horton-Smith, J. E. Spencer, D. Walz, S. C. Berridge, W. M. Bugg, K. Shmakov, A. W. Weidemann, C. Bula, K. T. McDonald, E. J. Prebys, C. Bamber, S. J. Boege, T. Koffas, T. Kotseroglou, A. C. Melissinos, D. D. Meyerhofer, D. A. Reis, and W. Ragg, ibid. 79, 1626 (1997); C. Bamber et al., Phys. Rev. D 60, 092004 (1999).

[11] W. P. Leemans et al., Nat. Phys. 2, 696 (2006); I. Blumenfeld et al., Nature (London) 445, 741 (2007); S. Kneip et al., Phys. Rev. Lett. 103, 035002 (2009).

[12] Y. Y. Lau et al., Phys. Plasmas 10, 2155 (2003); F. He, Y. Y. Lau, D. P. Umstadter, and R. Kowalczyk, Phys. Rev. Lett. 90, 055002 (2003); A. Zhidkov, J. Koga, A. Sasaki, and M. Uesaka, ibid. 88, 185002 (2002); S. V. Bulanov et al., Plasma Phys. Rep. 30, 196 (2004); N. M. Naumova, J. A. Nees, I. V. Sokolov, B. Hou, and G. A. Mourou, Phys. Rev. Lett. 92, 063902 (2004); N. Naumova, I. Sokolov, J. Nees, A. Maksimchuk, V. Yanovsky, and G. Mourou, ibid. 93, 195003 (2004); J. Nees et al., J. Mod. Opt. 52, 305 (2005); N. M. Naumova, J.
A. Nees, and G. A. Mourou, Phys. Plasmas 12, 056707 (2005).

[13] I. V. Sokolov, JETP 109, 207 (2009).

[14] I. V. Sokolov et al., Phys. Plasmas 16, 093115 (2009).

[15] J. D. Jackson, Classical Electrodynamics (Wiley, New York, 1999).

[16] A. I. Nikishov and V. I. Ritus, Sov. Phys. Usp. 13, 303 (1970); V. I. Ritus, J. Russ. Laser Res. 6, 497 (1985); V. I. Ritus, in Issues in Intense-Field Quantum Electrodynamics, edited by V. L. Ginzburg (Nova Science, Commack, 1987); see also the papers cited there: A. I. Nikishov and V. I. Ritus, Sov. Phys. JETP 19, 529 (1964); 19, 1191 (1964); 20, 757 (1965); N. B. Narozhny et al., ibid. 20, 622 (1965).

[17] V. N. Baier, V. M. Katkov, and V. M. Strakhovenko, Electromagnetic Processes at High Energies in Oriented Single Crystals (World Scientific, Singapore, 1998); V. Baier and V. Katkov, in Advanced Radiation Sources and Applications, edited by H. Wiedemann (Springer, Netherlands, 2006).

[18] A. Rousse et al., Phys. Rev. Lett. 93, 135005 (2004); S. Kiselev, A. Pukhov, and I. Kostyukov, ibid. 93, 135004 (2004).

[19] S. Kneip et al., Proc. SPIE 7359, 73590T (2009).

[20] J. L. Martins et al., Proc. SPIE 7359, 73590V (2009).

[21] D. Habs et al., Appl. Phys. B: Lasers Opt. 93, 349 (2008); D. Kiefer et al., Eur. Phys. J. D 55, 427 (2009).

[22] M. V. Galynsky and S. M. Sikach, Phys. Part. Nucl. 29, 469 (1998); L. Dongguo et al., Jpn. J. Appl. Phys. 42, 5376 (2003).

[23] A. Kh. Khokonov, M. Kh. Khokonov, and A. A. Kizdermishov, Tech. Phys. 47, 1413 (2002); E. Nerush and I. Kostyukov, Phys. Rev. E 75, 057401 (2007).

[24] T. Omori et al., Phys. Rev. Lett. 96, 114801 (2006).

[25] N. Naumova, T. Schlegel, V. T. Tikhonchuk, C. Labaune, I. V. Sokolov, and G. Mourou, Phys. Rev. Lett. 102, 025002 (2009); T. Schlegel et al., Phys. Plasmas 16, 083103 (2009); N. Naumova et al., Eur. Phys. J. D 55, 393 (2009).

[26] E. J. Moniz and D. H. Sharp, Phys. Rev. D 10, 1133 (1974); 15, 2850 (1977); A. Higuchi, ibid. 66, 105004 (2002).

[27] G. W. Ford and R. F. O'Connell, Phys. Lett. A 157, 217 (1991); R. F. O’Connell, ibid. 313, 491 (2003). 